\title{
G-protein-coupled receptor structure: what can we learn?
}

\section{Andrew B Tobin}

Address: Department of Cell Physiology and Pharmacology, University of Leicester, Hodgkin Building, Lancaster Road, Leicester LE1 9HN, UK

Email: tba@le.ac.uk

FI000 Biology Reports 2009, I:I I (doi: 10.34I0/BI-II)

The electronic version of this article is the complete one and can be found at: http://FI000.com/Reports/Biology/contents/I/I I

\begin{abstract}
The first, long-awaited, structures of non-visual G-protein-coupled receptors have provided important insights into the process of ligand binding and receptor activation. Here I explore the technological advances that enabled the resolution of the crystal structures of the $\beta_{1-}$ and $\beta_{2}$-adrenergic receptors, and review what we have learnt and what is still to be discovered.
\end{abstract}

\section{Introduction and context}

G-protein-coupled receptors (GPCRs) are a little like the famous red London buses; you wait years for a structure and when one at last arrives it is quickly followed by many. Seven years after the pioneering work on the rhodopsin structure by Palczewski et al. [1], the race for the first crystal structure of a non-visual GPCR has been won by research teams led by Brian Kobilka and Raymond Stevens working on the $\beta_{2}$-adrenergic receptor [2-4].

Why did it take so long and require such a huge international effort, and the development of numerous novel technologies, to arrive at a structure that was so highly prized by both academia and the pharmaceutical industry? This can essentially be explained by the fact that unlike rhodopsin, which forms a highly stable inactive conformational state, non-visual GPCRs adopt numerous conformational states even when they are considered to be inactive [5]. Such heterogeneity of conformation is not conducive to the formation of diffraction-quality crystals. Hence, ironically, the very characteristic of GPCRs that gives rise to complex pharmacology and an ability to regulate numerous biological processes, namely their conformational flexibility, is the primary characteristic that has hindered crystallisation and resolution of their structure. I will cover here the key factors that allowed the first structural determination of a non-visual GPCRs, and the major findings from these studies. There are also a number of excellent recent reviews to which the reader is directed for further analysis [6-10].

\section{Technological advances}

The $\beta_{2}$-adrenergic receptor, activated by the natural ligands adrenaline and noradrenaline, is of biomedical importance because of its cardiovascular and pulmonary function. However, it was the detailed pharmacological knowledge and biochemical expertise gathered over decades of investigation that made this receptor a more attractive proposition for crystallisation than any of the other $~ 800$ GPCRs. Technology developed more than 20 years ago for the purification of the $\beta$-adrenergic receptor from frog erythrocytes [11] - the first biochemical purification of a GPCR - provided the first step in the crystallisation process. Affinity-chromatography columns immobilising the receptor antagonist alprenolol enabled purification of the receptor such that nearly $100 \%$ of the receptors were functionally active [3]. In comparison, purification of the receptor using a resin-bound antibody against an amino (N)terminal FLAG epitope tag resulted in highly purified receptor, but less than $50 \%$ of the purified receptor was able to bind ligand [3]. Importantly, the ligand-affinitypurified receptor adopted a highly stable conformation if maintained in the presence of an antagonist. Under these conditions, solutions containing receptor concentrations of $\sim 50 \mathrm{mg} / \mathrm{ml}$ were stable for up to a week at room temperature [3]. 
The question for the crystallographic teams was which antagonist to use in the stabilisation of the receptor. Carazolol was chosen, as it has picomolar affinity for the receptor and very slow dissociation kinetics. Furthermore, carazolol is an inverse agonist and so is able to reduce the constitutive activity of the receptor by driving the equilibrium towards an inactive conformation. The proportion of receptors adopting a totally inactive conformation in the presence of carazolol would therefore be high, thus reducing the conformational heterogeneity that might otherwise prevent crystal formation.

It would seem that purification of functional receptor to high concentrations coupled with stabilisation of the receptor with antagonists would be sufficient to allow successful crystallisation. However, despite more than 2000 conditions being tested at different temperatures, diffraction-quality crystals were not obtained [3]. There still appeared to be conformational heterogeneity, and the receptor domains responsible for this were likely to be the flexible intracellular carboxy (C)-terminal tail and third intracellular loop [12]. In the two published structures of the $\beta_{2}$-adrenergic receptor, the teams led by Kobilka and Stevens tackled the flexibility of the C-terminal tail in the same way, namely by truncating this domain. However, each handled the problem of the flexibility of the third intracellular loop using different approaches. One was to use a conformation-specific antibody against the third intracellular loop to 'lock' the loop into a fixed conformation in a manner that did not affect the ligand-binding site (as judged by the pharmacological characteristics of ligand binding) [3]. This approach had previously been used to obtain the structure of a $\mathrm{K}^{+}$channel at a resolution of $2.0 \AA$ [13]. The other approach was to replace the third intracellular loop with T4 lysozyme, which is a highly ordered protein that readily forms crystals $[2,4]$. Both approaches effectively resulted in the same outcome, namely the removal of the most flexible regions of the receptor and their replacement with a highly ordered protein (antibody Fab fragment or T4 lysozyme), which in itself will form good crystals.

Another hurdle to overcome was the fact that GPCRs are highly hydrophobic proteins with seven helical domains that transverse the plasma membrane. It was therefore necessary to solubilise the receptor, and the detergent conditions used to achieve this must maintain the receptor in a native conformation and be conducive to crystallisation. This was achieved for the $\beta_{2}$-adrenergic receptor by using either a cholesterol-doped monolein lipid cubic phase $[2,4]$ or bicelles composed of the lipid dimyristoyl phosphatidylcholine and the detergent CHAPSO (DMPC/CHAPSO) [3].
Hence, by combining biochemical purification techniques, pharmacological stabilisation, protein engineering, antibody technology and lipid chemistry, crystals of the $\beta_{2}$-adrenergic receptor were obtained. These were, however, tiny - no more than $30 \mu \mathrm{m}$ across and $10 \mu \mathrm{m}$ thick $[2,4]$. Conventional X-ray beams are $50 \mu \mathrm{m}$ wide and were therefore unable to resolve these crystals, which were also readily destroyed in the beam. The final piece in the jigsaw was the application of high-intensity X-ray microbeams of less than $10 \mu \mathrm{m}$ diameter. The application of this technology finally revealed structures of the $\beta_{2}$-adrenergic receptor at $2.4 \AA[2,4]$ and 3.4/3.7 $\AA$ [3] resolution.

\section{Major recent advances Overall structure}

Until the publication of the $\beta_{2}$-adrenergic receptor structures, the conformation of GPCRs had been modelled on that of rhodopsin [14]. The first question, naturally, was how did the structure of the $\beta_{2}$-adrenergic receptor, which like rhodopsin is a class A GPCR, match the structure of rhodopsin? The answer is that overall there is a strong structural similarity between the two proteins. This is measured empirically by the root-meansquare (rms) deviation between the two structures, which for the transmembrane helical domains is $1.6 \AA$ and for the whole receptor is $2.3 \AA$. This is considered to represent very close structural similarity, as illustrated, for example, by a comparison of the closely related protein kinase catalytic domains of the enzymes PKC and PKA, which have an rms of $2.3 \AA$ but where the sequence identity is higher $(31 \%)$ than it is between $\beta_{2^{-}}$ adrenergic receptor and rhodopsin transmembrane domains (23\%) [8].

\section{Second extracellular loop}

Whereas the overall topography of the helical domains of the $\beta_{2}$-adrenergic receptor and rhodopsin are similar, the subtle differences seen between the two structures reveal something of the processes of ligand binding and receptor activation (Figure 1). In terms of ligand binding, the most striking feature is the position of the second extracellular loop (ECL2), which in rhodospin folds into a $\beta$ sheet that is buried within the helical transmembrane domains. Together with $\mathrm{N}$-terminal interactions, the buried ECL2 of rhodopsin forms a lid over the retinalbinding pocket protecting cis-retinal from hydrolysis. If such an arrangement were to occur in the $\beta_{2}$-adrenergic receptor, it would severely restrict access of ligands to the ligand-binding pocket. Instead, in the $\beta_{2}$-adrenergic receptor, the ECL2 forms a short helix that is constrained by two disulphide bonds. In this arrangement, the ECL2 of the $\beta_{2}$-adrenergic receptor is held away from the ligand-binding pocket. Furthermore, through the 
Figure I. Structures of the $\beta_{2}$-adrenergic receptor

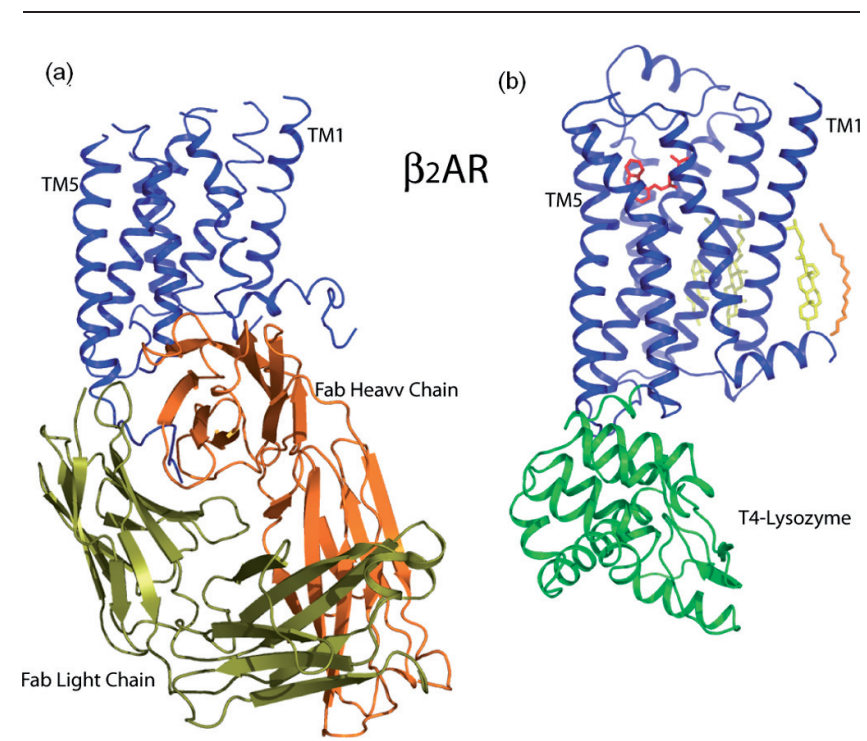

The structures of the $\beta_{2}$-adrenergic receptor determined by stabilisation using (a) the Fab fragment of a conformation-specific antibody ( $\beta_{2}$-adrenergic-Fab) and (b) the $\beta_{2}$-adrenergic receptor-T4 lysozyme fusion protein ( $\beta_{2}$-adrenergic-T4). The structures (a) and (b) were kindly created for this commentary by Brian Kobilka, Stanford University.

disulphide bonds and interactions with the first extracellular loop, the ECL2 forms a rigid structure that stabilises the core of the receptor. The recent resolution of the $\beta_{1}$-adrenergic receptor structure shows a similar conformation of the ECL2 [15], suggesting that this feature may be common among non-visual class A GPCRs (Figure 2).

\section{The ionic lock}

The second surprising structural feature is centred on the network of ionic and hydrogen-bond interactions between the conserved $(\mathrm{D} / \mathrm{E}) \mathrm{R}(\mathrm{Y} / \mathrm{W})$ amino-acid motif found at the cytoplasmic end of the third transmembrane domain (TM3) and residues at the cytoplasmic end of the sixth transmembrane domain (TM6). These interactions, known as the 'ionic lock', were observed in the rhodopsin structure [1] and are thought to stabilise the inactive conformation of the majority of class A receptors by linking the cytoplasmic ends of TM3 and TM6. Fluorescent spectroscopy techniques have found changes in the conformation of the ionic lock during activation of the receptor by agonists [16], supporting the notion that opening the structure of the ionic lock is important in receptor activation [5]. This notion is further supported by the recent crystal structure of ligand-free opsin, which adopts an active conformation and where the ionic lock is open [17]. Surprisingly, both structures of the $\beta_{2^{-}}$
Figure 2. Structure of the $\beta_{1}$-adrenergic receptor

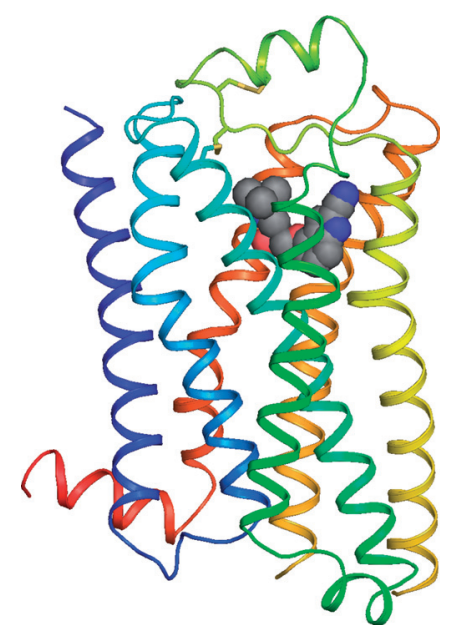

Shown is the recent structure of the $\beta_{1}$-adrenergic receptor. Figure 2 was kindly provided by Gerbhard Schertler, Laboratory of Molecular Biology, University of Cambridge.

adrenergic receptor revealed that the ionic lock was in the open conformation, a state previously associated with the active receptor. This was unexpected, as the receptor was crystallised in the presence of the inverse agonist carazolol, which should maintain it in an inactive conformation. It is possible that carazolol, which in fact only reduces $\beta_{2}$-adrenergic receptor basal activity by $50 \%$, does not maintain the receptor in a completely inactive conformation [3]. Therefore, the structure of the $\beta_{2^{-}}$ adrenergic receptor obtained in the presence of carazolol may represent a partially active conformation in which the ionic lock is open [3].

Recent resolution of the $\beta_{1}$-adrenergic receptor structure has, however, brought into question the original interpretation of the ionic lock. A thermostable mutant of the turkey $\beta_{1}$-adrenergic receptor was crystallised in the presence of the antagonist cyanopindolol [15]. Signalling assays established that the receptor had no basal activity and was therefore in a totally inactive state. The crystal structure of this receptor revealed that, like the $\beta_{2^{-}}$ adrenergic receptor, the ionic lock was in the open conformation. The authors of the $\beta_{1}$-adrenergic receptor structure suggest that the ionic lock is not important for the stabilisation of the inactive state of non-visual GPCRs and even suggest that this may also be the case for rhodopsin [15].

\section{The rotamer toggle}

The ionic lock is one of two molecular switches that have been proposed to operate during agonist activation of 
the receptor [5]. The second comprises structural rearrangements made possible by a series of conserved prolines within the transmembrane domains that introduce kinks into the helices. Of particular importance is the highly conserved proline kink in TM6, which in the $\beta_{2}$-adrenergic receptor is located at Pro288. The angle of the kink induced by Pro288 is dependent on interactions with aromatic residues close by (Trp286, Phe289, Phe290). Agonist binding promotes rotation of the aromatic residues and modulation of the angle of the proline kink in a process referred to as a 'rotamer toggle' $[18,19]$.

Although carazolol does not interact directly with Pro288, it does interact with the aromatic amino acids of the rotamer toggle, which adopt a rotameric state associated with the inactive receptor [2]. Importantly, the rotamer toggle is also associated with a series of water molecules that form a hydrogen-bonding network extending from the transmembrane helical bundle to the cytoplasmic surface [2]. It is possible that this network, which interacts with a number of highly conserved amino acids, is important in transmitting structural changes from the ligand-binding pocket to distal sites.

\section{Future directions}

The currently available structures of the $\beta_{1^{-}}$and $\beta_{2^{-}}$ adrenergic receptors represent enormous effort and ingenuity, and provide invaluable information regarding the nature of the receptor ligand-binding site and mechanisms of activation. However, they have serious limitations. First, the structures obtained are not of the native receptors but of highly engineered proteins. More important, however, is the fact that crystal structures will only give information about one conformation of the receptor. Because active receptor conformations are likely to be heterogeneous, with the receptor adopting multiple conformational states $[5,20]$, it may be that a crystal structure of an active receptor is not possible, or at least extremely difficult, to obtain. The first structure of an active GPCR - that of a photoactivated rhodopsin - has recently been published [21]. Nevertheless, other biophysical techniques that can more readily identify conformational change, such as site-directed spin labelling, may prove more informative [22]. Great hope has also been placed on the application of NMR to resolve the structural changes following agonist, antagonist and allosteric modulator binding [5].

So what can we expect from X-ray crystallography in the future? Certainly we can expect many more structures and more surprises. The recently reported structure of squid rhodopsin, for example, represents the first structure of a $\mathrm{G}_{\mathrm{q}}$-coupled GPCR [23]. The most pronounced feature of this receptor is that the transmembrane helices V and VI extend into the cytoplasm and combine with a helix in the extended C-terminal tail to form a rigid structure in the cytoplasm that has been called the helical dome [24]. This structure is thought to play a part in $\mathrm{G}_{\mathrm{q}}$-protein coupling. We will have to wait to see if similar structures are determined for vertebrate $\mathrm{G}_{\mathrm{q}}$-coupled GPCRs. What is certain, however, is that this pioneering work will continue to reveal how changes in the structure of this superfamily of transmembrane receptor proteins can mediate the plethora of biological responses that have been attributed to GPCR activity.

\section{Abbreviations}

ECL2, extracellular loop 2; GPCR, G-protein-coupled receptor; $\mathrm{PKA}$, protein kinase $\mathrm{A}$; $\mathrm{PKC}$, protein kinase $\mathrm{C}$; rms, root mean square; TM, transmembrane domain.

\section{Competing interests}

The author declares that he has no competing interests.

\section{Acknowledgements}

I would like to thank John Challiss and Adrian Butcher for reading the article and for suggestions. I am supported by a Senior Research Fellowship from the Wellcome Trust (047600).

\section{References}

I. Palczewski K, Kumasaka T, Hori T, Behnke CA, Motoshima H, Fox BA, Le Trong I, Teller DC, Okada T, Stenkamp RE, Yamamoto M, Miyano M: Crystal structure of rhodopsin: a $\mathbf{G}$ protein-coupled receptor. Science 2000, 289:739-45.

FI000 Factor 9.0 Exceptional

Evaluated by Jonathan Javitch 7 Jan 2002

2. Cherezov V, Rosenbaum DM, Hanson MA, Rasmussen SG, Thian FS, Kobilka TS, Choi HJ, Kuhn P, Weis WI, Kobilka BK, Stevens RC: High-resolution crystal structure of an engineered human beta ${ }_{2}$-adrenergic G protein-coupled receptor. Science 2007, 3 | 8: $1258-65$.

FI000 Factor II.0 Exceptional

Evaluated by Xiayang Qiu 3I Oct 2007, Mark von Zastrow 6 Nov 2007, Peter Artymiuk 27 Nov 2007, Patricia C Weber 3 Dec 2007, Ram Samudrala I4 Dec 2007, Petra Fromme 18 Dec 2007, Marc Caron 10 Jan 2008

3. Rasmussen SG, Choi HJ, Rosenbaum DM, Kobilka TS, Thian FS, Edwards PC, Burghammer M, Ratnala VR, Sanishvili R, Fischetti RF, Schertler GF, Weis WI, Kobilka BK. Crystal structure of the human beta 2 -adrenergic G-protein-coupled receptor. Nature 2007, 450:383-7.

FI000 Factor II.3 Exceptional

Evaluated by Leonardo Pardo 30 Oct 2007, Fred Dyda 16 Nov 2007, Carmen Dessauer 19 Nov 2007, Arthur Christopoulos 23 Nov 2007, Patricia C Weber 3 Dec 2007, lan Hall I3 Dec 2007, Marc Caron 10 Jan 2008, Stephan Nussberger 3 Mar 2008

4. Rosenbaum DM, Cherezov V, Hanson MA, Rasmussen SG, Thian FS, Kobilka TS, Choi HJ, Yao XJ, Weis WI, Stevens RC, Kobilka BK: GPCR engineering yields high-resolution structural insights 
into beta $a_{2}$-adrenergic receptor function. Science 2007, 3 I 8: 1266-73.

FI000 Factor 10.2 Exceptional

Evaluated by Xiayang Qiu 3I Oct 2007, Senyon Choe 19 Nov 2007, Marc Caron 10 Jan 2008, Thomas Baranski 26 Feb 2008

5. Kobilka BK, Deupi X: Conformational complexity of G-proteincoupled receptors. Trends Pharmacol Sci 2007, 28:397-406.

6. Kobilka B, Schertler GF: New G-protein-coupled receptor crystal structures: insights and limitations. Trends Pharmacol Sci 2008, 29:79-83.

7. Kobilka BK: G protein coupled receptor structure and activation. Biochim Biophys Acta 2007, I 768:794-807.

8. Lefkowitz RJ, Sun JP, Shukla AK: A crystal clear view of the beta2adrenergic receptor. Nat Biotechnol 2008, 26:|89-9|.

9. Shukla AK, Sun JP, Lefkowitz RJ: Crystallizing thinking about the beta $_{2}$-adrenergic receptor. Mol Pharmacol 2008, 73:1333-8.

10. Sprang SR: Structural biology: a receptor unlocked. Nature 2007, 450:355-6.

II. Caron MG, Srinivasan Y, Pitha J, Kociolek K, Lefkowitz RJ: Affinity chromatography of the beta-adrenergic receptor. J Biol Chem 1979, 254:2923-7.

12. Granier S, Kim S, Shafer AM, Ratnala VR, Fung J], Zare RN, Kobilka B: Structure and conformational changes in the C-terminal domain of the beta2-adrenoceptor: insights from fluorescence resonance energy transfer studies. J Biol Chem 2007, 282: | 3895-905.

13. Zhou Y, Morais-Cabral JH, Kaufman A, MacKinnon R: Chemistry of ion coordination and hydration revealed by $\mathbf{a} \mathrm{K}^{+}$channel-Fab complex at $2.0 \AA$ resolution. Nature 200I, 4l 4:43-8.

FI000 Factor 10.0 Exceptional

Evaluated by Gary Yellen 7 Nov 200I, Tom Rapoport I 5 Nov 200I, Christopher Miller 16 Nov 200I, Eaton E Lattman 4 Dec 2001

14. Ballesteros JA, Shi L, Javitch JA: Structural mimicry in G proteincoupled receptors: implications of the high-resolution structure of rhodopsin for structure-function analysis of rhodopsin-like receptors. Mol Pharmacol 2001, 60:1-19.

15. Warne T, Serrano-Vega MJ, Baker JG, Moukhametzianov R, Edwards PC, Henderson R, Leslie AG, Tate CG, Schertler GF: Structure of a betal-adrenergic G-protein-coupled receptor. Nature 2008, 454:486-91.

FI000 Factor 6.0 Must Read

Evaluated by Hao Wu I5 Sep 2008

16. Yao X, Parnot C, Deupi X, Ratnala VR, Swaminath G, Farrens D, Kobilka B: Coupling ligand structure to specific conformational switches in the beta2-adrenoceptor. Nat Chem Biol 2006, 2:417-22.

FI000 Factor 3.2 Recommended

Evaluated by Leonardo Pardo 25 Jul 2006, Arthur Christopoulos 27 Jul 2006

17. Park JH, Scheerer P, Hofmann KP, Choe HW, Ernst OP: Crystal structure of the ligand-free $G$-protein-coupled receptor opsin. Nature 2008, 454:|83-7.

FI000 Factor 8.3 Exceptional

Evaluated by Thomas Baranski 26 Jun 2008, Gordon Fain 18 Jul 2008, Stephen Sprang 21 Jul 2008, Hao Wu with Qian Yin 27 Jan 2009

18. Schwartz TW, Frimurer TM, Holst B, Rosenkilde MM, Elling CE: Molecular mechanism of 7TM receptor activation - a global toggle switch model. Annu Rev Pharmacol Toxicol 2006, 46:48I-5I9.

19. Shi L, Liapakis G, Xu R, Guarnieri F, Ballesteros JA, Javitch JA: Beta2 adrenergic receptor activation. Modulation of the proline kink in transmembrane 6 by a rotamer toggle switch. J Biol Chem 2002, 277:40989-96.

20. Kenakin T: Functional selectivity through protean and biased agonism: who steers the ship? Mol Pharmacol 2007, 72:1393-40I.

21. Salom D, Lodowski DT, Stenkamp RE, Le Trong I, Golczak M, Jastrzebska B, Harris T, Ballesteros JA, Palczewski K: Crystal structure of a photoactivated deprotonated intermediate of rhodopsin. Proc Natl Acad Sci U S A 2006, I03:16123-8.

FI000 Factor 6.4 Must Read

Evaluated by Jonathan Javitch 16 Nov 2006, Leonardo Pardo 23 Nov 2006

22. Altenbach C, Kusnetzow AK, Ernst OP, Hofmann KP, Hubbell WL: High-resolution distance mapping in rhodopsin reveals the pattern of helix movement due to activation. Proc Natl Acad Sci U S A 2008, 105:7439-44.

FI000 Factor 3.0 Recommended

Evaluated by David Cafiso 30 May 2008

23. Murakami M, Kouyama T: Crystal structure of squid rhodopsin. Nature 2008, 453:363-7.

FI000 Factor 8.0 Exceptional

Evaluated by Leonardo Pardo 27 May 2008, Arthur Christopoulos 4 Jun 2008

24. Schertler GF: Signal transduction: the rhodopsin story continued. Nature 2008, 453:292-3. 